and others (18\%). Grouped by themes, mapping was the most frequently used method, with $74 \%$ of articles limited to this approach to investigate a spatial pattern of injuries. Cluster detection and ecological analysis methods were applied less commonly, being used in $26 \%$ and $3 \%$ of articles, respectively. The kernal density estimation for point data and local indicators of spatial autocorelation for areal data were the most frequently used cluster detection methods.

Conclusion In the last two decades, many geospatial methods have been developed and applied in injury research, primarily to investigate road traffic injuries. The depth of investigations has been largely limited to basic mapping. Use of more advanced geospatial methods will help to better understand injury aetiology. Researchers should be encouraged to adopt these advanced methods in their future studies.

\section{STATE VARIATIONS IN MORTALITY FROM UNSPECIFIED UNINTENTIONAL INJURY AMONG AMERICANS AGED 65 YEARS AND OLDER, 1999-2013}

${ }^{1}$ Xunjie Cheng, ${ }^{2}$ Jie Yao, ${ }^{3}$ David C Schwebel, ${ }^{1 \#}$ Guoging Hu. ${ }^{1}$ Department of Epidemiology and Health Statistics, School of Public Health, Central South University; ${ }^{2}$ College of Basic Sciences and Humanities, Harbin Institute of Technology Shenzhen Graduate School; ${ }^{3}$ Department of Psychology, University of Alabama at Birmingham

\subsection{6/injuryprev-2016-042156.312}

Background Recent changes in unspecified unintentional injury mortality for the elderly by state remain unreported. This study aims to examine state variations in mortality from unspecified unintentional injury among Americans aged 65 +, 1999-2013.

Methods Using mortality rates from CDC's Web-based Injury Statistics Query and Reporting System (WISQARS ${ }^{\mathrm{TM}}$ ), we examined unspecified unintentional injury mortality for elderly individuals aged $65+$ from 1999 to 2013 by state. The proportion of unspecified unintentional injury was calculated to quantify the cause specificity of data. Linear regression examined the statistical significance of changes in proportion of unspecified unintentional injury from 1999 to 2013.

Results Of the 36 states with stable mortality rates, over 8 -fold differences were observed for both the mortality rates and the proportions of unspecified unintentional injury for Americans aged $65+$ during 1999-2013. 29 of the 36 states showed reductions in the proportion of unspecified unintentional injury, with Oklahoma (-89\%), Massachusetts $(-86 \%)$ and Oregon $(-81 \%)$ displaying the largest changes. The decrease in unspecified unintentional injury mortality was negatively correlated with the change in mortality from falls in 26 states and poisoning in 3 states, and positively correlated with mortality from suffocation in 15 states, motor vehicle traffic crashes in 12 states, and fire/ burn in 8 states.

Conclusions The cause specificity of elderly unintentional injury has improved for many states in the United States during 19992013. The improved cause specificity should be considered in trends analysis at the state level and comparisons between states for cause-specific injury mortality.

\section{RISK OF VIOLENCE-RELATED INJURY AND BURDEN TO SOCIETY IN THE LATIN AMERICAN AND CARIBBEAN REGION}

${ }^{1}$ Cheryl J Cherpitel, ${ }^{1} Y u$ Ye, ${ }^{2}$ Maristela Monteiro. ${ }^{1}$ Alcohol Research Group, Emeryville, CA, USA; ${ }^{2}$ Pan American Health Organisation, Washington DC, USA

\subsection{6/injuryprev-2016-042156.313}

Background Violence-related injury has been an important harmful consequence of drinking in the Latin American and Caribbean (LAC) region, but the risk at which drinking places the individual for violence-related injury or the burden this places on the population is unknown.

Methods A probability sample of 969 emergency room (ER) patients reporting a violence-related injury and arriving within six hours was analysed from 12 ERs in 9 countries in the LAC region. Dose-response relative risk (RR) of violence-related injury based on volume of consumption prior to the event is examined using pair-matched case-crossover fractional polynomial analysis of mean volume and alcohol attributable fraction (AAF) calculated from the RR and prevalence of drinking prior to injury in each volume category.

Results A dose-response relationship was observed with a six-fold increase in risk $(\mathrm{RR}=5.88)$ for less than two drinks prior to injury, increasing to a nine-fold increase $(R R=9.06)$ for those reporting more than 30 drinks. Risk was similar for males and females up to ten drinks, but higher for females over ten drinks. Risk was higher for those 30 and older compared to those younger at all volume levels, reaching a three-fold increase for 30 or more drinks $(\mathrm{RR}=4.78$ vs. 14.99$)$. Among all injuries related to violence, $33.56 \%$ were attributable to alcohol (population $A A F)$, and among those who reported drinking prior to injury, alcohol was an attributable factor in $87 \%$ (exposed $A A F$ ). AAF did not vary by age but was nearly three times larger for males (38.66\%) than for females (13.52\%), although exposed AAF did not differ.

Conclusions A dose-response relationship was found between the amount of alcohol consumed prior to the event and risk of violence-related injury, but risk was not uniform across gender or age. While females were at greater risk of injury than males at higher volume levels, lower prevalence of women drinking at higher levels contributed to overall lower AAF for women.

\section{INJURY RECIDIVISTS IN A DEFINED POPULATION OCCURRENCE AND CHARACTERISTICS}

'Børge Ytterstad, ${ }^{2}$ Thomas Gressnes. 'University of Tromsø, Norway; ${ }^{2}$ Harstad University College, Norway

\subsection{6/injuryprev-2016-042156.314}

Background Studies on injury recidivism in Pubmed are mainly based solely on admissions to emergency departments for certain injury types. For tailoring prevention programs, such studies may aid identification of target groups for interventions. To our knowledge, population based studies on the full spectre of injury recidivism in a community have not been published so far.

Methods The Harstad Injury Prevention Studies have relied on data from a continuously recorded injury data base (IDB) since 1985. Coding was done in the Nordic system. From 2010 a unique identifier variable (ID $=11$ digit person number) was added to the IDB after permission from the data inspectorate. The present work is based on injuries treated at Harstad Hospital (admitted and outpatient) containing this ID. Some person 